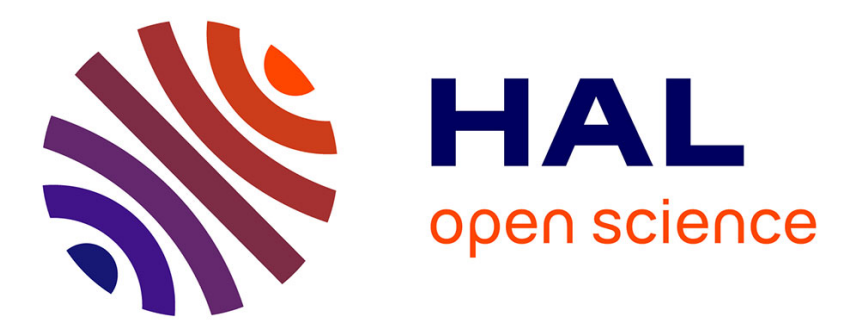

\title{
Stability of LTI systems with distributed sensors and aperiodic sampling
}

Christophe Fiter, Taki-Eddine Korabi, Lucien Etienne, Laurentiu Hetel

\section{To cite this version:}

Christophe Fiter, Taki-Eddine Korabi, Lucien Etienne, Laurentiu Hetel. Stability of LTI systems with distributed sensors and aperiodic sampling. Control subject to Computational and Communication Constraints: Current Challenges, Lecture Notes in Control and Information Sciences, Springer, 2018. hal-01689253

\section{HAL Id: hal-01689253 https://hal.inria.fr/hal-01689253}

Submitted on 9 Jul 2018

HAL is a multi-disciplinary open access archive for the deposit and dissemination of scientific research documents, whether they are published or not. The documents may come from teaching and research institutions in France or abroad, or from public or private research centers.
L'archive ouverte pluridisciplinaire HAL, est destinée au dépôt et à la diffusion de documents scientifiques de niveau recherche, publiés ou non, émanant des établissements d'enseignement et de recherche français ou étrangers, des laboratoires publics ou privés. 


\title{
Stability of LTI systems with distributed sensors and aperiodic sampling
}

Christophe Fiter, Taki-Eddine Korabi, Lucien Etienne, and Laurentiu Hetel

\begin{abstract}
This chapter is dedicated to the stability analysis of sampled-data linear time invariant systems with asynchronous sensors and aperiodic sampling. The study is performed using an input/output interconnection modelling, and tools from the robust control theory. Two approaches are presented. One is based on the small gain theorem, while the other is based on the dissipativity theory. Tractable stability criteria that allow an estimation of the Maximal Admissible Sampling Period are obtained for both approaches. Finally, experimental results performed on an inverted pendulum benchmark are presented. They confirm the applicability of both approaches and allow for some comparisons between both results.
\end{abstract}

\section{Introduction}

Networked Control Systems (NCSs) are often required to share limited communication and computation resources, which may lead to fluctuations of the sampling interval [Chen and Francis (1993)] [Zhang et al. (2001)]. These variations bring up

Christophe Fiter

Centre de Recherche en Informatique, Signal, et Automatique de Lille (CRIStAL, CNRS UMR 9189) - Université de Lille 1, 59650 Villeneuve d'Ascq, France

e-mail: christophe.fitereuniv-lillel.fr

Taki-Eddine Korabi

CRIStAL, CNRS UMR 9189 - École Centrale de Lille, 59650 Villeneuve d'Ascq, France

e-mail: taki-inhiste@hotmail.fr

Lucien Etienne

Non-A team at INRIA Lille, 59650 Villeneuve d'Ascq, France

e-mail: lucien.etiennedinria.fr

Laurentiu Hetel

CRIStAL, CNRS UMR 9189 - École Centrale de Lille, 59650 Villeneuve d'Ascq, France

e-mail: laurentiu.heteldec-lille.fr 
new challenges from the control theory point of view, since they may be sources of unstability. In order to cope with this phenomenon, several researches have recently been conducted to analyse the stability of systems with arbitrarily time-varying sampling intervals (see the survey [Hetel et al. (2017)]). Time-delay approaches ([Fridman et al. (2004)] [Fridman (2010)] [Seuret (2012)]), hybrid systems approaches ([Karafyllis and Kravaris (2004)] [Nesic et al. (2009)]), discrete-time approaches ([Cloosterman et al. (2010)] [Hetel et al. (2006)] [Fiter et al. (2012)]), and input/output stability approaches ([Kao and Lincoln (2004)] [Mirkin (2007)] [Fujioka (2009)] [Omran et al. (2014)]) have been used to address this problem.

Most of the studies have concerned the stability analysis of systems with one sensor measuring the whole system's state. A problem that must be addressed in practical applications is how to guarantee the stability of a system with multiple sensors. In the case of periodic sampling, this problem has been solved a while ago in the context of multirate systems [Araki and Yamamoto (1986)]. However, measurements are usually aperiodic and asynchronous. To the best of our knowledge, this problem has not yet been studied in the literature.

In this work, we propose to tackle this issue in the case of Linear Time Invariant (LTI) systems, using two approaches from the robust control theory. Both approaches are based on a modelling of the system as an interconnection between a continuous-time (sampling-free) closed-loop system and an operator representing the sampling effects. This new model can be seen as an extension of the one proposed in [Fujioka (2009)] in the single sensor case. The first result, based on the small gain theorem, provides simple numerical tools to design an overall upperbound on the sampling intervals that guarantees the system's stability. The second result, which is based on the dissipativity theory, allows through the use of Linear Matrix Inequalities (LMIs) to compute an upper-bound on the sampling interval for each and every sensor, that guarantee the system's stability.

The chapter is constructed as follows. First, in Section 2, we present the system under study and formulate the problem. In Section 3, we propose the new modelling of the system that will be used for the whole analysis. Then, in Sections 4 and 5, we present the two stability analysis approaches, based respectively on the small gain theorem and on the dissipativity theory. Finally, in Section 6, we illustrate our results with experiments on an inverted pendulum benchmark, before concluding in Section 7.

Notations: $I_{M}$ denotes the set $\{1, \ldots, M\}$. The Euclidean norm of a vector $x$ is denoted by $|x|$, and its $\mathscr{L}_{2}$ norm is denoted by $\|x\|_{L_{2}}$. The $\mathscr{L}_{2}$ induced norm of an operator $\mathrm{G}$ is denoted by $\|G\|_{L_{2}-L_{2}}$. A function $\beta: \mathbb{R}_{\geq 0} \rightarrow \mathbb{R}_{\geq 0}$ is said to be of class $\mathscr{K}$ if it is continuous, zero at zero and strictly increasing. It is said to be of class $\mathscr{K}_{\infty}$ if it is of class $\mathscr{K}$, and it is unbounded. A function $\beta: \mathbb{R}_{\geq 0} \times \mathbb{R}_{\geq 0} \rightarrow \mathbb{R}_{\geq 0}$ is said to be of class $\mathscr{K} \mathscr{L}$ if $\beta(., t)$ is of class $\mathscr{K}$ for each $t \geq 0$, and $\beta(s,$.$) is non-increasing$ and satisfies $\lim _{t \rightarrow \infty} \beta(s, t)=0$ for each $s \geq 0$. We use $\operatorname{diag}\left(N_{1}, \cdots, N_{M}\right)$ to denote a block diagonal matrix which contains the blocks $N_{i}\left(i \in I_{M}\right)$ on its diagonal, and zeros everywhere else. 


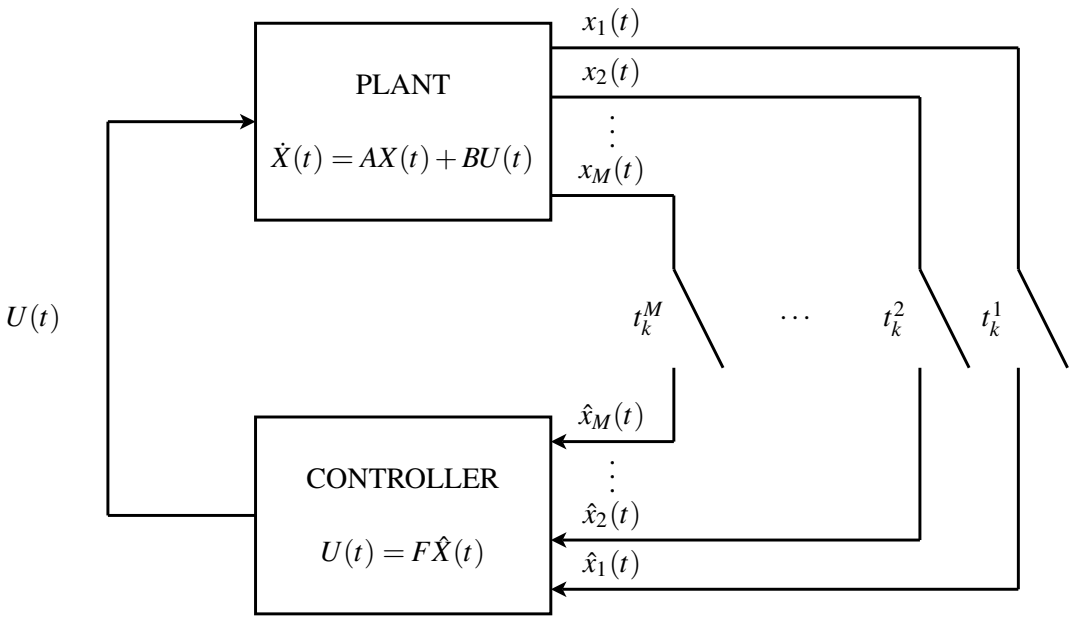

Fig. 1 System description

\section{Problem formulation}

We consider the linear time-invariant (LTI) system with distributed sensors presented in Figure 1.

This system is mathematically defined by

$$
\begin{aligned}
& \dot{X}(t)=A X(t)+B U(t), \forall t \geq 0, \\
& X(0)=X_{0} \in \mathbb{R}^{n},
\end{aligned}
$$

with $X \in \mathbb{R}^{n}$ the system state, $X_{0} \in \mathbb{R}^{n}$ the initial state, $U \in \mathbb{R}^{m}$ the input, and $A$ and $B$ matrices of appropriate dimensions.

We consider that the state $X$ is divided into $M$ sub-parts $x_{i}, i \in I_{M}$, each linked to one sensor:

$$
X(t)=\left[x_{1}^{T}(t) \ldots x_{M}^{T}(t)\right]^{T},
$$

with $x_{i} \in \mathbb{R}^{n_{i}}$ and $\sum_{i=1}^{M} n_{i}=n$.

These sub-parts of the state are transmitted to the controller in an asynchronous manner. For each of them, we consider a monotonously increasing sampling sequence with bounded sampling intervals

$$
\sigma^{i}=\left\{t_{k}^{i}\right\}_{k \in \mathbb{N}} \text { with } t_{k+1}^{i}-t_{k}^{i} \in\left(0, h_{i}\right]
$$

We assume that $t_{0}^{i}=0$ for all $i \in I_{M}$.

The sampled-state is denoted $\hat{X}(t)=\left[\hat{x}_{1}^{T}(t) \ldots \hat{x}_{M}^{T}(t)\right]^{T}$, with

$$
\hat{x}_{i}(t)=x_{i}\left(t_{k}^{i}\right), \forall t \in\left[t_{k}^{i}, t_{k+1}^{i}\right), k \in \mathbb{N}, i \in I_{M}
$$


The control is considered as a linear state-feedback using the known, sampled version of the state:

$$
U(t)=F \hat{X}(t),
$$

where $F \in \mathbb{R}^{m \times n}$ is a given feedback gain.

The aim of this work is to design methods for checking the stability of system (1),(2),(3),(4).

\section{System modelling}

In this section, adapting the approach proposed in [Kao and Lincoln (2004)] in the single sensor case, and further used in [Mirkin (2007)], [Fujioka (2009)], and [Omran et al. (2014)], we remodel the system as an interconnection between a continuous-time closed-loop system and an operator representing the sampling effect on all the sub-parts of the system's state.

We define the sampling system error

$$
E(t)=\hat{X}(t)-X(t)=\left[e_{1}^{T}(t) \ldots e_{M}^{T}(t)\right]^{T},
$$

where, for all $i \in I_{M}$,

$$
\begin{aligned}
e_{i}(t) & =\hat{x}_{i}(t)-x_{i}(t), \forall t \geq 0, \\
& =x_{i}\left(t_{k}^{i}\right)-x_{i}(t), \forall t \in\left[t_{k}^{i}, t_{k+1}^{i}\right), k \in \mathbb{N} .
\end{aligned}
$$

Let us remark that the sampling error can be described as a reset integrator

$$
e_{i}(t)=-\int_{t_{k}^{i}}^{t} \dot{x}_{i}(\theta) d \theta, \forall t \in\left[t_{k}^{i}, t_{k+1}^{i}\right), k \in \mathbb{N} .
$$

For each $i \in I_{M}$ we define the operator

$$
\begin{gathered}
\Delta_{i}: \mathscr{L}_{2 e}^{n_{i}}[0, \infty) \mapsto \mathscr{L}_{2 e}^{n_{i}}[0, \infty) \\
z \mapsto \Delta_{i} z \\
\left(\Delta_{i} z\right)(t)=-\int_{t_{k}^{i}}^{t} z(\theta) d \theta, \forall i \in I_{M}, \forall t \in\left[t_{k}^{i}, t_{k+1}^{i}\right) .
\end{gathered}
$$

Finally, the system (1),(2),(3),(4) can be represented by the interconnection of $G: E \mapsto Y$, the LTI system described by

$$
G:\left\{\begin{array}{l}
\dot{X}(t)=A_{\mathrm{cl}} X(t)+B_{\mathrm{cl}} E(t), \\
Y(t)=C_{\mathrm{cl}} X(t)+D_{\mathrm{cl}} E(t),
\end{array}\right.
$$




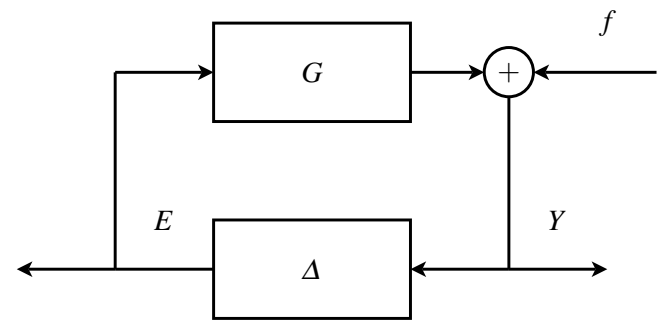

Fig. 2 System's representation as an interconnected system

$$
\text { with }
$$

$A_{\mathrm{cl}}=C_{\mathrm{cl}}=A+B F, B_{\mathrm{cl}}=D_{\mathrm{cl}}=B F, Y(t)=\left[y_{1}^{T}(t) \ldots y_{M}^{T}(t)\right]^{T}$, and the operator $\Delta: Y \mapsto E$ defined by

$$
\Delta: E=\Delta Y
$$

with $\Delta=\operatorname{diag}\left(\Delta_{1}, \cdots, \Delta_{M}\right)$.

Here, $A_{\mathrm{cl}}$ represents the nominal (closed-loop) state matrix, and $E$ the samplinginduced error described by operator $\Delta$ in (7).

This representation of the system as an interconnection (8), (9), will be used throughout the rest of the chapter for the stability analysis.

Note that this interconnection can also be rewritten so as to explicitely see the influence of the initial state $x_{0}$. Indeed, let $\mathrm{G}: \mathscr{L}_{2}^{\infty}[0, \infty) \mapsto \mathscr{L}_{2}^{\infty}[0, \infty)$ be the linear operator described by the transfer function

$$
\hat{G}(s)=C_{\mathrm{cl}}\left(s I-A_{\mathrm{cl}}\right)^{-1} B_{\mathrm{cl}}+D_{\mathrm{cl}}=s\left(s I-A_{\mathrm{cl}}\right)^{-1} B_{\mathrm{cl}} .
$$

If we consider the free response of system (8)

$$
f(t)=A_{\mathrm{cl}} e^{A_{\mathrm{cl}} t} x_{0}, \forall t \geq 0,
$$

we can rewrite the system as the interconnection

$$
\begin{aligned}
& Y=G E+f, \\
& E=\Delta Y .
\end{aligned}
$$

This interconnection is presented in Figure 2.

\section{Small gain approach}

The first stability approach we consider is based on the small gain theorem [Khalil (2002)]. Before presenting the main result of this approach, we need to provide a technical lemma. 
Lemma 1. The $\mathscr{L}_{2}$ induced norm of the operator $\Delta$ is upper-bounded by $h_{\max }$, where $h_{\text {max }}=\max _{i \in I_{M}}\left\{h_{i}\right\}$.

Proof. Using (6) for all $i \in I_{M}$ and $t \in\left[t_{k}^{i}, t_{k+1}^{i}\right), k \in \mathbb{N}$, we consider

$$
e_{i}(t)=-\int_{t_{k}^{i}}^{t} y_{i}(\theta) d \theta, \forall t \in\left[t_{k}^{i}, t_{k+1}^{i}\right), k \in \mathbb{N} .
$$

Then, using Jensen's inequality [Gu et al. (2003)], we get

$$
\begin{aligned}
e_{i}^{T}(t) e_{i}(t) & =\left(\int_{t_{k}^{i}}^{t} y_{i}(\theta) d \theta\right)^{T}\left(\int_{t_{k}^{i}}^{t} y_{i}(\theta) d \theta\right) \\
& \leq\left(t-t_{k}^{i}\right)\left(\int_{t_{k}^{i}}^{t} y_{i}^{T}(\theta) y_{i}(\theta) d \theta\right) \\
& \leq h_{i}\left(\int_{t_{k}^{i}}^{t} y_{i}^{T}(\theta) y_{i}(\theta) d \theta\right)
\end{aligned}
$$

Applying further inequalities, we can compute an upper-bound on $\left\|e_{i}\right\|_{L_{2}}$ :

$$
\begin{aligned}
\left\|e_{i}\right\|_{L_{2}}^{2} & =\int_{0}^{\infty} e_{i}^{T}(t) e_{i}(t) d t \\
& =\sum_{k=0}^{\infty} \int_{t_{k}^{i}}^{t_{k+1}^{i}} e_{i}^{T}(t) e_{i}(t) d t \\
& \leq \sum_{k=0}^{\infty} \int_{t_{k}^{i}}^{t_{k+1}^{i}} h_{i} \int_{t_{k}^{i}}^{t} y_{i}^{T}(\theta) y_{i}(\theta) d \theta d t \\
& \leq h_{i} \sum_{k=0}^{\infty} \int_{t_{k}^{i}}^{t_{k+1}^{i}} \int_{t_{k}^{i}}^{t_{k+1}^{i}} y_{i}^{T}(\theta) y_{i}(\theta) d \theta d t \\
& =h_{i} \sum_{k=0}^{\infty}\left(t_{k+1}^{i}-t_{k}^{i}\right) \int_{t_{k}^{i}}^{t_{k+1}^{i}} y_{i}^{T}(\theta) y_{i}(\theta) d \theta \\
& \leq h_{i}^{2} \sum_{k=0}^{\infty} \int_{t_{k}^{i}}^{t_{k+1}^{i}} y_{i}^{T}(\theta) y_{i}(\theta) d \theta \\
& =h_{i}^{2} \int_{0}^{\infty} y_{i}^{T}(\theta) y_{i}(\theta) d \theta \\
& =h_{i}^{2}\left\|_{i}\right\|_{L_{2}}^{2} .
\end{aligned}
$$

We have found that

$$
\left\|e_{i}\right\|_{L_{2}}^{2} \leq h_{i}^{2}\left\|y_{i}\right\|_{L_{2}}^{2}, \forall i \in I_{M}
$$

From this equation, considering the sampling error $E$ as described in (5), and using some majorations, we may also compute an upper-bound on $\|E\|_{L_{2}}$ : 


$$
\begin{aligned}
\|E\|_{L_{2}}^{2} & =\int_{0}^{\infty} E^{T}(t) E(t) d t, \\
& =\int_{0}^{\infty} \sum_{i=1}^{M} e_{i}^{T}(t) e_{i}(t) d t, \\
& =\sum_{i=1}^{M} \int_{0}^{\infty} e_{i}^{T}(t) e_{i}(t) d t, \\
& \leq \sum_{i=1}^{M} h_{i}^{2} \int_{0}^{\infty} y_{i}^{T}(\theta) y_{i}(\theta) d \theta, \\
& \leq h_{\max }^{2} \sum_{i=1}^{M} \int_{0}^{\infty} y_{i}^{T}(\theta) y_{i}(\theta) d \theta \\
& =h_{\max }^{2} \int_{0}^{\infty} \sum_{i=1}^{M} y_{i}^{T}(\theta) y_{i}(\theta) d \theta, \\
& =h_{\max }^{2} \int_{0}^{\infty} Y^{T}(\theta) Y(\theta) d \theta, \\
& =h_{\max }^{2}\|Y\|_{L_{2}}^{2} .
\end{aligned}
$$

Therefore, we have obtained that

$$
\|E\|_{L_{2}}^{2} \leq h_{\max }^{2}\|Y\|_{L_{2}}^{2},
$$

which ends the proof.

Before giving the main result of this section, we recall the following definition [Khalil (2002)].

Definition 1 ( $\mathscr{L}_{2}$-stability). A linear system $\mathbf{F}$ is said to be finite-gain $\mathscr{L}_{2}$-stable from $w$ to $\mathbf{F} w$ with an induced gain less than $\gamma$ if $\mathbf{F}$ is a linear operator from $\mathscr{L}_{2}$ to $\mathscr{L}_{2}$ and if there exist positive real constants $\gamma$ and $\xi$ such that for all $w \in \mathscr{L}_{2}$,

$$
\|\mathbf{F} w\|_{L_{2}} \leq \gamma\|w\|_{L_{2}}+\xi
$$

Theorem 1. The interconnection (10) is $\mathscr{L}_{2}$-stable if $\|G\|_{\infty}<\frac{1}{h_{\max }}$.

Proof. We use the small gain theorem to provide the stability condition. A direct consequence of this theorem is the fact that if

$$
\|G\|_{L_{2}-L_{2}}\|\Delta\|_{L_{2}-L_{2}}<1,
$$

the interconnection (10) is $\mathscr{L}_{2}$-stable.

Since G is linear, its $\mathscr{L}_{2}$-induced norm can be calculated [Zhou et al. (1996)] using the $H_{\infty}$ norm of its transfer function:

$$
\|G\|_{L_{2}-L_{2}}=\|G\|_{\infty}:=\sup _{w \in \mathbb{R}} \bar{\sigma}(\hat{G}(j w)) .
$$


Since $\|\Delta\|_{L_{2}} \leq h_{\max }$, according to Lemma 1 , the interconnection (10) is $\mathscr{L}_{2-}$ stable if

$$
\|G\|_{L_{2}-L_{2}}=\|G\|_{\infty}<\frac{1}{h_{\max }} .
$$

Remark 1 . The condition proposed in the previous theorem can be easily implemented in Matlab. It depends solely on the system, and on the overall upper-bound on the sampling intervals $h_{\max }$. However, this approach does not make it possible to take into account the difference in the upper-bounds of each sensor $\left(\left\{h_{1}, \cdots, h_{M}\right\}\right)$ in the analysis. In the next section, we propose an approach that solves this problem.

\section{Dissipativity based approach}

In this section, we propose another stability approach inpired by the dissipativity theory [Willems (1972)] for the interconnected system (8), (9). We first start by providing a few tools and definitions.

Let us define a sequence $\gamma$ that includes all the sampling sequences $\sigma^{i}=\left\{t_{k}^{i}\right\}_{k \in \mathbb{N}}$, $i \in I_{M}$, in chronological order:

$$
\gamma=\left\{t_{s}\right\}_{s \in \mathbb{N}}, \text { with } t_{0}=0 \text { and } t_{s+1}=\min _{i \in I_{M}, k \in \mathbb{N}}\left\{t_{k}^{i}: t_{k}^{i}>t_{s}\right\}, \forall s \in \mathbb{N} .
$$

For any $i \in I_{M}$ and $s \in \mathbb{N}$, let us also define the coefficient

$$
k_{i, s}=\max \left\{k \in \mathbb{N}: t_{k}^{i} \leq t_{s}\right\},
$$

which represents the index of the last sample sent to the controller before the time $t_{s}$, by sensor $i$. Intuitively, the time $t_{k_{i, s}}^{i}$, for $i \in I_{M}$ and $s \in \mathbb{N}$ represents the last time before $t_{s}$ that sensor $i$ sent a measure to the controller.

It is important to remark that for all $s \in \mathbb{N}, t_{s+1}-t_{s} \in\left(0, h_{\min }\right]$, where $h_{\text {min }}=$ $\min _{i \in I_{M}}\left\{h_{i}\right\}$.

In order to give a better understanding of the notations that were just introduced, we show in Figure 3 an example of construction of a sequence $\gamma$ in the case of a system with 2 sensors. The value of the scalars $k_{i, s}$ obtained from these sequences are shown in table 1 .

Before giving the main results, we recall the following stability definition:

Definition 2 (GUAS). The equilibrium point $X=0$ of system (1), (2), (3), (4) is globally uniformly asymptotically stable (GUAS) if there exists a class $\mathscr{K} \mathscr{L}$ function $\beta(.,$.$) , such that$ 


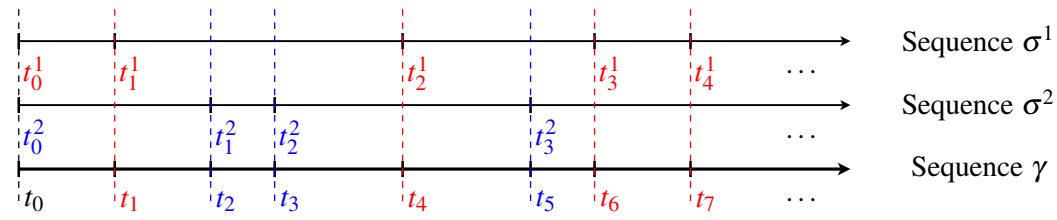

Fig. 3 An example of sampling sequences with 2 sensors

\begin{tabular}{|l|c|c|c|c|c|c|c|c|c|}
\hline & $s=0$ & $s=1$ & $s=2$ & $s=3$ & $s=4$ & $s=5$ & $s=6$ & $s=7$ & $\cdots$ \\
\hline$i=1$ (sensor 1) & 0 & 1 & 1 & 1 & 2 & 2 & 3 & 4 & $\cdots$ \\
\hline$i=2$ (sensor 2) & 0 & 0 & 1 & 2 & 2 & 3 & 3 & 3 & $\cdots$ \\
\hline
\end{tabular}

Table 1 Value of the coefficients $k_{i, s}$ in the example

$$
|X(t)| \leq \beta\left(\left|X\left(t_{0}\right)\right|, t-t_{0}\right), \forall t \geq t_{0}, \forall X\left(t_{0}\right) \in \mathbb{R}^{n} .
$$

Theorem 2. Consider the sampled-data system (1), (2), (3), (4) and the equivalent representation (8), (9). Consider the definition of $e_{i}(t)$ in (6). Assume that:

1. There exist $M$ continuous functions $\mathscr{S}_{i}\left(y_{i}, e_{i}\right)$ which satisfy the integral property

$$
\int_{t_{k}^{i}}^{t} \mathscr{S}_{i}\left(y_{i}(\theta), e_{i}(\theta)\right) d \theta \leq 0, \forall t \in\left[t_{k}^{i}, t_{k+1}^{i}\right), k \in \mathbb{N}, i \in I_{M} .
$$

2. There exist a differentiable positive define function $V: \mathbb{R}^{n} \rightarrow \mathbb{R}$ and class $\mathscr{K}$ functions $\beta_{1}$ and $\beta_{2}$ verifying

$$
\beta_{1}(|X|) \leq V(X) \leq \beta_{2}(|X|), \forall X \in \mathbb{R}^{n} .
$$

3. There exists a scalar $\alpha>0$ such that

$$
\begin{aligned}
& \dot{V}(X(t))+\alpha V(X(t)) \leq \sum_{i=1}^{M} e^{-\alpha \tau_{i}(t)} \mathscr{S}_{i}\left(y_{i}(t), e_{i}(t)\right) \\
& \forall t \in\left[t_{s}, t_{s+1}\right), \forall s \in \mathbb{N}, \text { with } \tau_{i}(t)=t-t_{k_{i, s}^{i}}, \text { where } k_{i, s} \text { is defined in (13). }
\end{aligned}
$$

Then the equilibrium point $X=0$ of the system (1),(2),(3),(4) is GUAS.

Proof. Consider $s \in \mathbb{N}$ and $t \in\left[t_{s}, t_{s+1}\right)$. From (17), we have

$$
\mathrm{e}^{\alpha t}\left(\dot{V}(X(t))+\alpha V(X(t)) \leq \sum_{i=1}^{M} \mathrm{e}^{\alpha t_{k_{i, s}^{i}}^{i}} \mathscr{S}_{i}\left(y_{i}(t), e_{i}(t)\right) .\right.
$$

By integrating this equation on the interval $\left[t_{s}, t\right]$, we get

$$
\mathrm{e}^{\alpha t} V(X(t))-\mathrm{e}^{\alpha t_{s}} V\left(X\left(t_{s}\right)\right) \leq \sum_{i=1}^{M} \mathrm{e}^{\alpha t_{i, s}^{i}} \int_{t_{s}}^{t} \mathscr{S}_{i}\left(y_{i}(\theta), e_{i}(\theta)\right) d \theta
$$


which leads to

$$
V(X(t)) \leq \mathrm{e}^{-\alpha\left(t-t_{s}\right)} V\left(X\left(t_{s}\right)\right)+\mathrm{e}^{-\alpha t} \sum_{i=1}^{M} \mathrm{e}^{\alpha t_{i, s}^{i}} \int_{t_{s}}^{t} \mathscr{S}_{i}\left(y_{i}(\theta), e_{i}(\theta)\right) d \theta .
$$

Therefore, we can see that for any $s \in \mathbb{N}$, we have

$$
V\left(X\left(t_{s+1}\right)\right) \leq \mathrm{e}^{-\alpha\left(t_{s+1}-t_{s}\right)} V\left(X\left(t_{s}\right)\right)+\mathrm{e}^{-\alpha t_{s+1}} \sum_{i=1}^{M} \mathrm{e}^{\alpha t_{i, s}^{i}} \int_{t_{s}}^{t_{s+1}} \mathscr{S}_{i}\left(y_{i}(\theta), e_{i}(\theta)\right) d \theta
$$

Replacing $V\left(X\left(t_{s}\right)\right)$ in (18) by its expression (19) leads to

$$
\begin{aligned}
V(X(t)) \leq & \mathrm{e}^{-\alpha\left(t-t_{s}\right)}\left[\mathrm{e}^{-\alpha\left(t_{s}-t_{s-1}\right)} V\left(X\left(t_{s-1}\right)\right)\right. \\
& \left.+\mathrm{e}^{-\alpha t_{s}} \sum_{i=1}^{M} \mathrm{e}^{\alpha t_{i, s-1}^{i}} \int_{t_{s-1}}^{t_{s}} \mathscr{S}_{i}\left(y_{i}(\theta), e_{i}(\theta)\right) d \theta\right] \\
& +\mathrm{e}^{-\alpha t} \sum_{i=1}^{M} \mathrm{e}^{\alpha t_{i, s}^{i}} \int_{t_{s}}^{t} \mathscr{S}_{i}\left(y_{i}(\theta), e_{i}(\theta)\right) d \theta
\end{aligned}
$$

and thus

$$
\begin{aligned}
V(X(t)) \leq & \mathrm{e}^{-\alpha\left(t-t_{s-1}\right)} V\left(X\left(t_{s-1}\right)\right)+\mathrm{e}^{-\alpha t} \sum_{i=1}^{M}\left[\mathrm{e}^{\alpha t_{i, s-1}^{i}} \int_{t_{s-1}}^{t_{s}} \mathscr{S}_{i}\left(y_{i}(\theta), e_{i}(\theta)\right) d \theta\right. \\
& \left.+\mathrm{e}^{\alpha t_{k_{i, s}}^{i}} \int_{t_{s}}^{t} \mathscr{S}_{i}\left(y_{i}(\theta), e_{i}(\theta)\right) d \theta\right] .
\end{aligned}
$$

Then, by recursivity, replacing $V\left(X\left(t_{s-1}\right)\right)$ in (20) by its expression (19) and so on, we can show that

$$
\begin{aligned}
V(X(t)) \leq & \mathrm{e}^{-\alpha\left(t-t_{0}\right)} V\left(X\left(t_{0}\right)\right)+\mathrm{e}^{-\alpha t} \sum_{i=1}^{M}\left[\left(\sum_{j=1}^{s} \mathrm{e}^{\alpha t_{k_{i, j-1}}^{i}} \int_{t_{j-1}}^{t_{j}} \mathscr{S}_{i}\left(y_{i}(\theta), e_{i}(\theta)\right) d \theta\right)\right. \\
& \left.+\mathrm{e}^{\alpha t_{k_{i, s}}^{i}} \int_{t_{s}}^{t} \mathscr{S}_{i}\left(y_{i}(\boldsymbol{\theta}), e_{i}(\boldsymbol{\theta})\right) d \theta\right] .
\end{aligned}
$$

Let us now consider some $k \in \mathbb{N}$ and $i \in I_{M}$. By construction, there exist positive integers $s_{1}$ and $s_{2}$, with $s_{1}<s_{2}$, such that $t_{k}^{i}=t_{s_{1}}$ and $t_{k+1}^{i}=t_{s_{2}}$. From the definition of elements $t_{s}$ and $k_{i, s}$ in (12) and (13) respectively (see also Figure 3 and Table 1 for an example), one can see that for any $\bar{s} \in\left\{s_{1}, s_{1}+1, \cdots, s_{2}-1\right\}, k_{i, \bar{s}}=k$, and thus $t_{k_{i, \bar{s}}}^{i}=t_{k}^{i}$. Therefore, using this property, we can rewrite (21) as 


$$
\begin{aligned}
V(X(t)) \leq & \mathrm{e}^{-\alpha\left(t-t_{0}\right)} V\left(X\left(t_{0}\right)\right)+\mathrm{e}^{-\alpha t} \sum_{i=1}^{M}\left[\left(\sum_{k=0}^{k_{i, s}-1} \mathrm{e}^{\alpha t_{k}^{i}} \int_{t_{k}^{i}}^{t_{k+1}^{i}} \mathscr{S}_{i}\left(y_{i}(\theta), e_{i}(\theta)\right) d \theta\right)\right. \\
& \left.+\mathrm{e}^{\alpha t_{k_{i, s}}^{i}} \int_{t_{k_{i, s}^{i}}^{i}}^{t} \mathscr{S}_{i}\left(y_{i}(\theta), e_{i}(\theta)\right) d \theta\right] .
\end{aligned}
$$

Then, using assumption (15), one can see that all the integral terms are negative, and thus one gets

$$
V(X(t)) \leq \mathrm{e}^{-\alpha\left(t-t_{0}\right)} V\left(X\left(t_{0}\right)\right)
$$

Finally, assumption (16) leads to

$$
\begin{aligned}
|X(t)| & \leq \beta_{1}^{-1}\left(V\left(X\left(t_{0}\right)\right) e^{-\alpha\left(t-t_{0}\right)}\right), \\
& \leq \beta_{1}^{-1}\left(\beta_{2}\left(\left|X\left(t_{0}\right)\right|\right) e^{-\alpha\left(t-t_{0}\right)}\right), \\
& :=\beta\left(\left|X\left(t_{0}\right)\right|, t-t_{0}\right), \forall t>t_{0},
\end{aligned}
$$

which concludes the proof.

Theorem 2 is based on the existence of $M$ functions $\mathscr{S}_{i}, i \in I_{M}$, satisfying assumption (15). In the following, we show that such functions exist.

First we start by providing a technical lemma.

Lemma 2. Consider the set of operators $\Delta_{i}$ defined in (7) for any $z_{i} \in \mathscr{L}_{2 e}^{n_{i}}$ and $t \in\left[t_{k}^{i}, t_{k+1}^{i}\right), k \in \mathbb{N}$. We have the following inequality

$$
\int_{t_{k}^{i}}^{t}\left(\Delta_{i} z_{i}\right)(\rho)^{T}\left(\Delta_{i} z_{i}\right)(\rho)-h_{i}^{2} z_{i}^{T}(\rho) z_{i}(\rho) d \rho \leq 0 .
$$

It follows that the $M$ functions $\mathscr{S}_{i}$ such that

$$
\mathscr{S}_{i}\left(y_{i}(\theta), e_{i}(\theta)\right)=e_{i}^{T}(\theta) e_{i}(\theta)-h_{i}^{2} y_{i}^{T}(\theta) y_{i}(\theta),
$$

with $e_{i}=\Delta_{i} y_{i}$, as defined in (7), satisfy the assumption (15).

Proof. Using the same steps as in the proof of Lemma 1, we can show that

$$
\left(\Delta_{i} z_{i}\right)^{T}(\rho)\left(\Delta_{i} z_{i}\right)(\rho) \leq h_{i}\left(\int_{t_{k}^{i}}^{\rho} z_{i}^{T}(\theta) z_{i}(\theta) d \theta\right), \forall \rho \in\left[t_{k}^{i}, t_{k+1}^{i}\right], k \in \mathbb{N} .
$$

Therefore we have, for all $t \in\left[t_{k}^{i}, t_{k+1}^{i}\right]$ and $k \in \mathbb{N}$,

$$
\begin{aligned}
\int_{t_{k}^{i}}^{t}\left(\Delta_{i} z_{i}\right)^{T}(\rho)\left(\Delta_{i} z_{i}\right)(\rho) d \rho & \leq \int_{t_{k}^{i}}^{t} h_{i} \int_{t_{k}^{i}}^{\rho} z_{i}^{T}(\theta) z_{i}(\theta) d \theta d \rho \\
& \leq h_{i} \int_{t_{k}^{i}}^{t} \int_{t_{k}^{i}}^{t} z_{i}^{T}(\theta) z_{i}(\theta) d \theta d \rho \\
& =h_{i}\left(t-t_{k}^{i}\right) \int_{t_{k}^{i}}^{t} z_{i}^{T}(\theta) z_{i}(\theta) d \theta
\end{aligned}
$$




$$
\leq h_{i}^{2} \int_{t_{k}^{i}}^{t} z_{i}^{T}(\theta) z_{i}(\theta) d \theta
$$

which ends the proof.

Lemma 2 proves the existence of functions $\mathscr{S}_{i}$ satisfying condition (15) in Theorem 2. In the following, we show the existence of a more general, parameterdependent, class of functions $\mathscr{S}_{i}$ satisfying that condition. The idea is to use a scaling matrix $R_{i}$ that gives an additionnal degree of liberty in the choice of the $\mathscr{S}_{i}$ functions. This will be useful later to reduce the conservatism of the stability conditions.

Lemma 3. Consider the set of operators $\Delta_{i}$ in (7), (9). Then for any $z_{i} \in \mathscr{L}_{2 e}^{n_{i}}$, $0<R_{i}^{T}=R_{i} \in \mathbb{R}^{m \times m}$ we have the following inequality

$\mathscr{N}(t)=\int_{t_{k}^{i}}^{t}\left[\left(\Delta_{i} z_{i}\right)^{T}(\rho) R_{i}\left(\Delta_{i} z_{i}\right)(\rho)-h_{i}^{2} z_{i}^{T}(\rho) R_{i} z_{i}(\rho)\right] d \rho \leq 0, \forall t \in\left[t_{k}^{i}, t_{k+1}^{i}\right), k \in \mathbb{N}$.

It follows that the M functions $\mathscr{S}_{i}$ such that

$$
\mathscr{S}_{i}\left(y_{i}(\theta), e_{i}(\theta)\right)=e_{i}^{T}(\theta) R_{i} e_{i}(\theta)-h_{i}^{2} y_{i}^{T}(\theta) R_{i} y_{i}(\theta),
$$

with $e_{i}=\Delta_{i} y_{i}$, as defined in (7), satisfy the assumption (15).

Proof. First of all, we note that since $R_{i}^{T}=R_{i}>0$, then there exists $U_{i} \in \mathbb{R}^{n \times n}$ such that $R_{i}=U_{i}^{T} U_{i}$. Therefore, for any $t \in\left[t_{k}^{i}, t_{k+1}^{i}\right), k \in \mathbb{N}$ we have

$$
\mathscr{N}(t)=\int_{t_{k}^{i}}^{t}\left[\left(U_{i}\left(\Delta_{i} z_{i}\right)(\rho)\right)^{T}\left(U_{i}\left(\Delta_{i} z_{i}\right)(\rho)\right)-h_{i}^{2}\left(U_{i} y_{i}(\rho)\right)^{T}\left(U_{i} y_{i}(\rho)\right)\right] d \rho .
$$

From (7) we can see that $U_{i}\left(\Delta_{i} z_{i}\right)=\Delta_{i}\left(U_{i} z_{i}\right)$. Then

$$
\mathscr{N}(t)=\int_{t_{k}^{i}}^{t}\left[\left(\Delta_{i}\left(U_{i} z_{i}\right)(\rho)\right)^{T}\left(\Delta_{i}\left(U_{i} z_{i}\right)(\rho)\right)-h_{i}^{2}\left(U_{i} y_{i}(\rho)\right)^{T}\left(U_{i} y_{i}(\rho)\right)\right] d \rho .
$$

Therefore, we can write

$$
\mathscr{N}(t)=\int_{t_{k}^{i}}^{t}\left[\left(\Delta_{i} \psi_{i}(\rho)\right)^{T}\left(\Delta_{i} \psi_{i}(\rho)\right)-h_{i}^{2} \psi_{i}^{T}(\rho) \psi_{i}(\rho)\right] d \rho,
$$

with $\psi_{i}(\rho)=U_{i} z_{i}(\rho)$, which can be seen to be negative from Lemma 2 .

We have now shown that the $\mathscr{S}_{i}$ functions satisfying the assumption (15) exist. The next step consists on showing that we can obtain a function $V$ which satisfies conditions (16) and (17), based on the $\mathscr{S}_{i}$ functions in Lemma 3.

Remark 2. The $\mathscr{S}_{i}$ functions presented in Lemmas 2 and 3 were inspired by the ones proposed in [Omran et al. (2014)], where it was studied the stability of bilinear systems with aperiodic sampling and a single sensor measuring the whole state. 


\subsection{Numerical criteria}

The goal of this section is to provide tractable LMI conditions that guarantee the stability conditions of Theorem 2, using Lemma 3. The main result is as follows.

Theorem 3. If there exist symmetric positive definite matrices $R_{i} \in \mathbb{R}^{n_{i} \times n_{i}}\left(i \in I_{M}\right)$, $P \in \mathbb{R}^{n \times n}$, and a scalar $\alpha>0$ such that the LMIs

$$
\left[\begin{array}{cc}
A_{c l}^{T} P+P A_{c l}+\alpha P+A_{c l}^{T} \bar{L}(\lambda) A_{c l} & P B_{c l}+A_{c l}^{T} \bar{L}(\lambda) B_{c l} \\
* & -\bar{R}(\lambda)+B_{c l}^{T} \bar{L}(\lambda) B_{c l}
\end{array}\right] \leq 0
$$

are satisfied for all $\lambda=\left[\lambda_{1}, \cdots, \lambda_{M}\right]^{T} \in\{0,1\}^{M}$, with

$$
\bar{R}(\lambda)=\operatorname{diag}\left(e^{-\alpha h_{1} \lambda_{1}} R_{1}, \cdots, e^{-\alpha h_{M} \lambda_{M}} R_{M}\right)
$$

and

$$
\bar{L}(\lambda)=\operatorname{diag}\left(e^{-\alpha h_{1} \lambda_{1}} h_{1}^{2} R_{1}, \cdots, e^{-\alpha h_{M} \lambda_{M}} h_{M}^{2} R_{M}\right),
$$

then the equilibrium $X=0$ of system (1), (2), (3), (4) is GUAS.

Proof. The goal is to provide sufficient conditions for the existence of functions $\mathrm{V}$ and $\mathscr{S}_{i}$ that satisfy the conditions of Theorem 2 . Consider a quadratic function $V(X(t))=X^{T}(t) P X(t)$ and the representation of the system in (8) where $Y(t)=$ $A_{\mathrm{cl}} X(t)+B_{\mathrm{cl}} E(t)$. Multiplying the LMIs (27) by $\left(X^{T}(t), E^{T}(t)\right)$ on the left and by its transpose on the right implies that

$$
\begin{aligned}
& 2 X^{T}(t) P\left(A_{\mathrm{cl}} X(t)+B_{\mathrm{cl}} E(t)\right)+\alpha X^{T}(t) P X(t) \\
& \leq E^{T}(t) \bar{R}(\lambda) E(t)-Y^{T}(t) \bar{L}(\lambda) Y(t), \forall \lambda \in\{0,1\}^{M} .
\end{aligned}
$$

Let us now define

$$
\tilde{R}(t)=\operatorname{diag}\left(\mathrm{e}^{-\alpha \tau_{1}(t)} R_{1}, \cdots, \mathrm{e}^{-\alpha \tau_{M}(t)} R_{M}\right),
$$

and

$$
\tilde{L}(t)=\operatorname{diag}\left(\mathrm{e}^{-\alpha \tau_{1}(t)} h_{1}^{2} R_{1}, \cdots, \mathrm{e}^{-\alpha \tau_{M}(t)} h_{M}^{2} R_{M}\right) .
$$

Taking into account the fact that for any $t \in\left[t_{s}, t_{s+1}\right), s \in \mathbb{N}$, one has $\tau_{i}(t)=t-t_{k_{i, s}}^{i} \in$ $\left[0, h_{i}\right)$ for all $i \in I_{M}$, it is clear that $\tilde{L}(t) \in \operatorname{conv}\left\{\tilde{L}(\lambda): \lambda \in\{0,1\}^{M}\right\}$ and $\tilde{R}(t) \in$ $\operatorname{conv}\left\{\bar{R}(\lambda): \lambda \in\{0,1\}^{M}\right\}$. Then, since (28) is satisfied, by convexity we obtain

$$
\begin{aligned}
& 2 X^{T}(t) P\left(A_{\mathrm{cl}} X(t)+B_{\mathrm{cl}} E(t)\right)+\alpha X^{T}(t) P X(t) \\
& \leq E^{T}(t) \tilde{R}(t) E(t)-Y^{T}(t) \tilde{L}(t) Y(t), \forall t \in\left[t_{s}, t_{s+1}\right), s \in \mathbb{N} .
\end{aligned}
$$

This leads to

$$
\begin{aligned}
& 2 X^{T}(t) P\left(A_{\mathrm{cl}} X(t)+B_{\mathrm{cl}} E(t)\right)+\alpha X^{T}(t) P X(t) \\
& \leq \sum_{i=1}^{M} \mathrm{e}^{-\alpha \tau_{i}(t)}\left(e_{i}^{T}(t) R_{i} e_{i}(t)-h_{i}^{2} y_{i}^{T}(t) R_{i} y_{i}(t)\right)
\end{aligned}
$$


which is equivalent to

$$
\dot{V}(X(t))+\alpha V(X(t)) \leq \sum_{i=1}^{M} \mathrm{e}^{-\alpha \tau_{i}(t)} \mathscr{S}_{i}\left(y_{i}(t), e_{i}(t)\right), \forall t \in\left[t_{s}, t_{s+1}\right), s \in \mathbb{N},
$$

with the functions $\mathscr{S}_{i}$ defined in Lemma 3 that satisfy (15). Therefore, assumption (17) in Theorem 2 is satisfied.

Furthermore, we can show that using Lemma 4.3 in [Khalil (2002)] for a quadratic function $V(X(t))=X^{T}(t) P X(t)$, assumption (16) is satisfied with funtions $\beta_{1}$ and $\beta_{2}$ defined such that $\beta_{1}(|X|)=\lambda_{\min }(P)|X|^{2}$ and $\beta_{2}(|X|)=\lambda_{\max }(P)|X|^{2}$.

Therefore, we can see that the quadratic function $V$, and the $\mathscr{S}_{i}$ functions in Lemma 3 satisfy all the conditions of Theorem 2, if the LMIs in (27) are feasible, which ends the proof.

Remark 3. Theorem 3 provides tractable LMI conditions for LTI systems with distributed, asynchronous sensors. Unlike the result obtained in the previous section, in Theorem 1, which depends on the overall upper-bound $h_{\max }=\max _{i \in I_{M}}\left\{h_{i}\right\}$, the conditions of Theorem depend explicitely on each sensor's sampling upper-bound $h_{i}, i \in I_{M}$.

\section{Validation of the results on an inverted pendulum benchmark}

In this section, we show some experimentations performed on an inverted pendulum benchmark, using the results from the previous sections. The system is obviously nonlinear, so we will use for analysis a linearisation of the model around its unstable equilibrium point (upper position).

\subsection{Benchmark description}

The inverted pendulum (see Figure 4), consists of a cart which is driven by a linear motor and a pendulum. The pendulum is fixed and left free on the cart. The system also consists of two sensors: the first one measures the linear position of the cart where the second one measures the angular position of the pendulum. An estimation of the linear and angular velocity is calculated using a filtered derivative. The communication between the system and the calculator (computer) is assured by a Dspace card. The control task is performed using SIMULINK and the ControlDesk software, which can allow us to see in real time the informations coming from the sensors and also to send commands to the linear motor. 

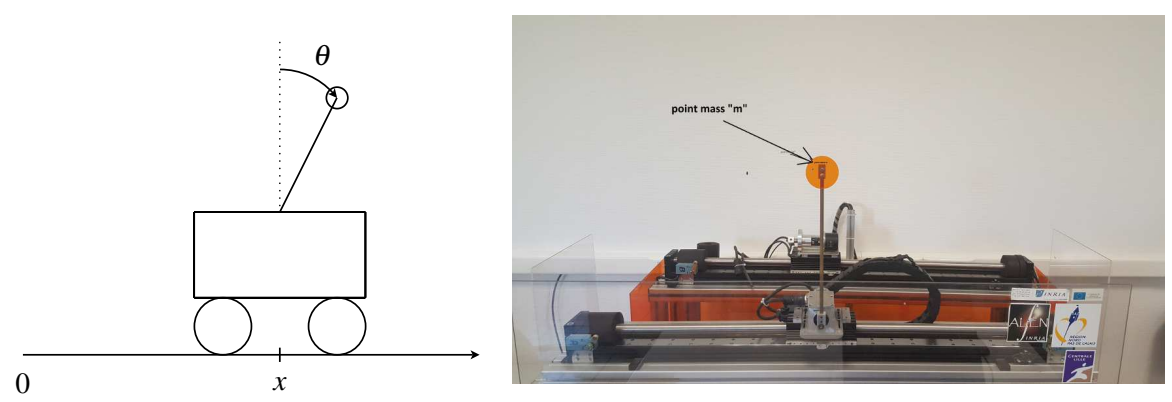

Fig. 4 Inverted pendulum at CRIStAL

\subsection{Dynamic model}

The Lagrangian of the system is represented by the following equation

$$
L=\frac{1}{2} M v_{1}^{2}+\frac{1}{2} m v_{2}^{2}-m g l \cos \theta
$$

where $\theta$ stands for the angular displacement measured from the equilibrium position. $\mathrm{M}$ and $\mathrm{m}$ represent the mass of the cart and and the point mass respectively. $v_{1}$ and $v_{2}$ are respectively the velocity of the cart and the velocity of the point mass $m$. $l$ is the length of the rod, and $g$ is the gravitational constant. After replacing $v_{1}$ and $v_{2}$ by their expression, and simplifying the result, the Lagrangian is given by

$$
L=\frac{1}{2}(M+m) \dot{x}^{2}-m l \dot{x} \dot{\theta} \cos \theta+\frac{1}{2} m l^{2} \dot{\theta}^{2}-m g l \cos \theta,
$$

where $x$ denotes the cart's position. Using the equation of Euler-Lagrange, one can easily calculate the equations of motion of the system

$$
\begin{aligned}
& (M+m) \ddot{x}-m l \sin \theta \dot{\theta}^{2}+m l \cos \theta \ddot{\theta}=N, \\
& m l^{2} \ddot{\theta}+m l \cos \theta \ddot{x}-m g l \sin \theta=0,
\end{aligned}
$$

where $N=\alpha U$ is the force exercised on the cart. The matrix representation of these equations is

$$
\left[\begin{array}{c}
\dot{x} \\
\ddot{x} \\
\dot{\theta} \\
\ddot{\theta}
\end{array}\right]=\left[\begin{array}{c}
\dot{x} \\
\frac{\left(-m g l \cos \theta+m l^{2} \dot{\theta}^{2}\right) \sin \theta}{l\left(M+m \sin ^{2} \theta\right)} \\
\dot{\theta} \\
\frac{\left((M+m) g-m l \cos \theta \dot{\theta}^{2}\right) \sin \theta}{l\left(M+m \sin ^{2} \theta\right)}
\end{array}\right]+\left[\begin{array}{c}
0 \\
\frac{l}{l\left(M+m \sin ^{2} \theta\right)} \\
0 \\
\frac{-\cos \theta}{l\left(M+m \sin ^{2} \theta\right)}
\end{array}\right] N .
$$

We will consider the linearisation of (35) at the upper position: 


$$
\left[\begin{array}{c}
\dot{x} \\
\ddot{x} \\
\dot{\theta} \\
\ddot{\theta}
\end{array}\right]=\left[\begin{array}{cccr}
0 & 1 & 0 & 0 \\
0 & 0 & \frac{-m g}{M} & 0 \\
0 & 0 & 0 & 1 \\
0 & 0 & \frac{(m+M) g}{M l} & 0
\end{array}\right]\left[\begin{array}{c}
x \\
\dot{x} \\
\theta \\
\dot{\theta}
\end{array}\right]+\left[\begin{array}{c}
0 \\
\frac{\alpha}{M} \\
0 \\
\frac{-\alpha}{M l}
\end{array}\right] U .
$$

The parameters describing the linear model (36) are given in Table 2.

\begin{tabular}{|c|c|c|}
\hline Parameters & Values & Characteristics \\
\hline$M$ & $3.9249 \mathrm{Kg}$ & mass of the cart \\
\hline$m$ & $0.2047 \mathrm{Kg}$ & mass of the m point \\
\hline$l$ & $0.2302 \mathrm{~m}$ & length of the rod \\
\hline$g$ & $9.81 \mathrm{~N} / \mathrm{kg}$ & gravitational constant \\
\hline$\alpha$ & $25.3 \mathrm{~N} / \mathrm{V}$ & motor's gain \\
\hline
\end{tabular}

Table 2 Inverted pendulum benchmark parameters

\subsection{Theoretical results}

In the following, we consider a feedback control gain (4)

$$
F=\left[\begin{array}{lll}
5.825 & 5.88324 .945 .140
\end{array}\right]
$$

which was obtained using a placement of the poles $\{-100,-2+2 i,-2-2 i,-2\}$.

We consider the case of $M=2$ sensors, one measuring $x_{1}=[x \dot{x}]^{T}$, and one measuring $x_{2}=\left[\begin{array}{ll}\theta & \dot{\theta}\end{array}\right]^{T}$.

Using the result from the small gain approach (Theorem 1), we can show that the interconnected system (10) is $\mathscr{L}_{2}$-stable if $h_{\text {max }}=\max _{i \in I_{M}}\left\{h_{i}\right\}<\frac{1}{\|G\|_{\infty}}=1.3 \mathrm{~ms}$. Note that although this approach allows to design an admissible upper-bound $h_{\max }$ for the sampling intervals, it does not make it possible to take into account the difference in the upper-bounds of the two sensors $\left(h_{1}\right.$ and $\left.h_{2}\right)$ in the analysis.

Using the the LMI result from the dissipativity based approach (Theorem 3), it is possible to design admissible upper-bounds on the sampling intervals for each sensor. The obtained stability domain is shown in Figure 5.

On top of allowing the design of each sensor's admissible sampling interval upper-bound separately, this approach seems to be much less conservative than the previous one. 


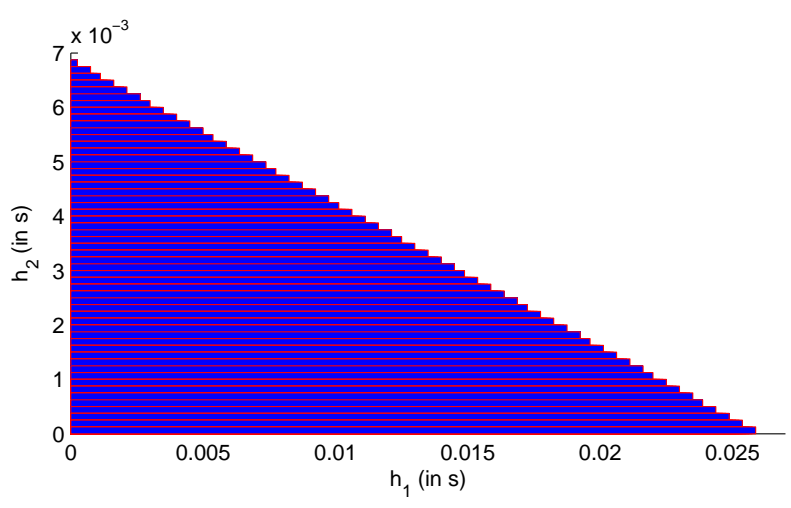

Fig. 5 Stability domain obtained with the dissipativity based approach
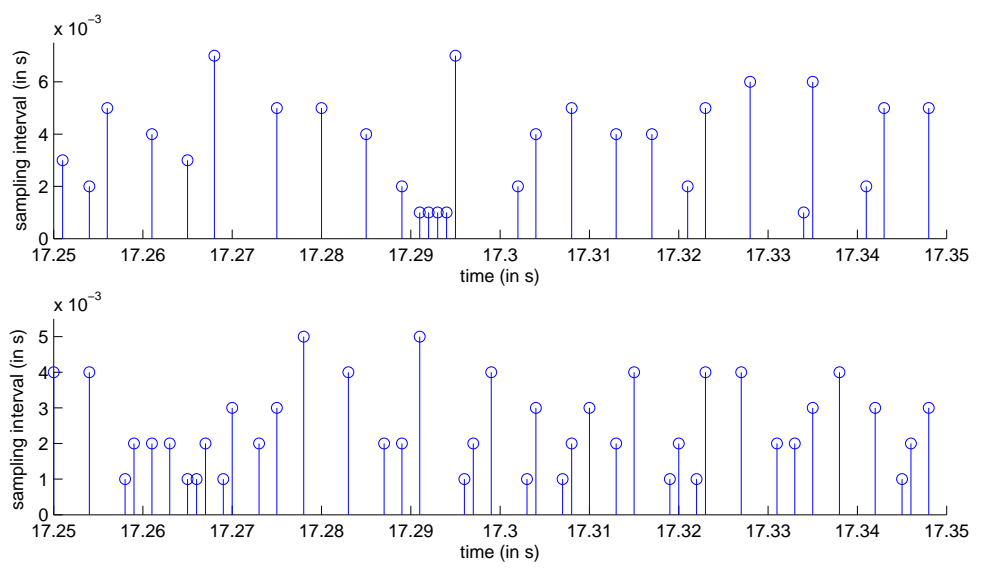

Fig. 6 Profile of the sampling of $x$ and $\dot{x}$ (on top), and of $\theta$ and $\dot{\theta}$ (at the bottom)

\subsection{Experimental results}

In this section, we present the experimentations performed using our benchmark. The results have been obtained using randomly varying sampling intervals with upper-bounds $h_{1}=7 m s$ and $h_{2}=5 m s$ (see Figure 6 to see the profile of the sampling intervals). Note that these upper-bounds are within the stability domain obtained with the dissipativity approach, which is presented in Figure 5.

The evolution of the position $x$ and angular position $\theta$ is presented in Figure 7 , where large perturbations have been introduced at $t=3.1 \mathrm{~s}, t=16.4 \mathrm{~s}$, and $t=25.9 \mathrm{~s}$, by acting on the pendulum manually. One can see that the pendulum stabilizes even in the presence of sampling intervals variations and asynchronicity of both sensors. The absence of oscillating patterns can be explained by the presence of dry friction. 

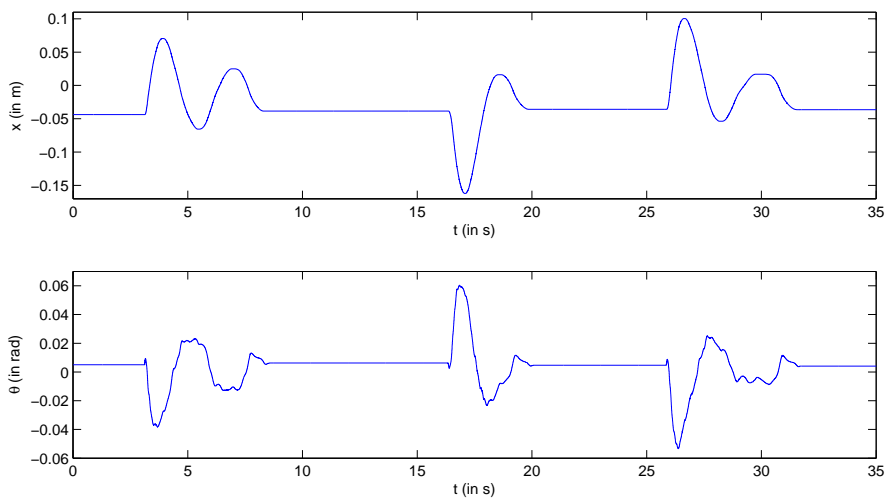

Fig. 7 Evolution of the position $x$ (top) and the angular position $\theta$ (bottom) of the inverted pendulum suject to perturbations for $h_{1}=7 \mathrm{~ms}$ and $h_{2}=5 \mathrm{~ms}$

\section{Conclusion}

This chapter was dedicated to the stability analysis of LTI sampled-data systems with asynchronous sensors and aperiodic sampling. The study is based on tools from the robust control theory, and on a modelling of the system as an input/output interconnection between an operator, representing the sampling error, and a continuoustime (i.e. sampling-free) closed-loop system. Two approaches have been presented. The first one is based on the small gain theorem, and provides a tractable estimation of the overall (with respect to all the sensors) maximum allowable sampling period. The second approach is based on the dissipativity theory. It provides simple numerical stability criterion using LMIs. An advantage of that approach compared to the previous one is that it allows for computing an estimation of the maximum allowable sampling for each and every sensor of the system. Experimentations have been performed on an inverted pendulum benchmark, and confirm the applicability of both approaches. The results seem to indicate that the dissipativity based approach is less conservative than small gain based one. In further studies, it would be interesting to consider additional phenomena in one study, such as delays or quantization.

\section{References}

Araki, M., Yamamoto, K. (1986). Multivariable multirate sampled-data systems: state-space description, transfer characteristics and Nyquist criterion. IEEE Transactions on Automatic Control, 31(2), 145-154.

Chen, T., Francis, B. A. (1993). Optimal sampled-data control systems. Springer. 
Cloosterman, M.-B.-G., Hetel, L., van de Wouw, N., Heemels, W.-P.-M.-H., Daafouz, J., Nijmeijer, H. (2010). Controller synthesis for networked control systems. Automatica, 46(10), 1584-1594.

Fiter, C., Hetel, L., Perruquetti, W., Richard, J.-P. (2012). A state dependent sampling for linear state feedback. Automatica, 48(8), 1860-1867.

Fridman, E., Seuret, A. Richard, J. P. (2004). Robust sampled-data stabilization of linear systems: an input delay approach. Automatica, 40(8), 1441-1446.

Fridman, E. (2010). A refined input delay approach to sampled-data control. Automatica, 46(2), 421-427.

Fujioka H. (2009). Stability analysis of systems with aperiodic sample-and-hold devices. Automatica, 45(3), 771-775.

Gu, K., Kharitonov, V., Chen, J. (2003). Stability of time-delay systems. Boston: Birkhauser.

Hetel, L., Daafouz, J., Iung, C. (2006). Stabilization of arbitrary switched linear systems with unknown time-varying delays. IEEE Transactions on Automatic Control, 51(10), 1668-1674.

Hetel, L., Fiter, C., Omran, H., Seuret, A., Fridman, E., Richard, J.-P., Niculescu, S.-I. (2017). Recent developments on the stability of systems with aperiodic sampling: An overview. Automatica, 76, 309-335.

Kao, C. Y., Lincoln, B. (2004). Simple stability criteria for systems with timevarying delays. Automatica, 40(8), 1429-1434.

Karafyllis, I., Kravaris, C. (2009). Global stability results for systems under sampled-data control. International Journal of Robust and Nonlinear Control, 19(10), 1105-1128.

Khalil, H. (2002) Nonlinear systems, 3rd edition. New Jewsey, Prentice Hall 9.

Mirkin, L. (2007). Some remarks on the use of time-varying delay to model sampleand-hold circuits. IEEE Transactions on Automatic Control, 52(6), 1109-1112.

Nesic, D., Teel, A., Carnevale, D. (2009). Explicit computation of the sampling period in emulation of controllers for nonlinear sampled-data systems. IEEE Transactions on Automatic Control, 54(3), 619-624.

Omran, H., Hetel, L., Richard, J. P., Lamnabhi-Lagarrigue, F. (2014). Stability analysis of bilinear systems under aperiodic sampled-data control. Automatica, 50(4), 1288-1295.

Seuret, A. (2012). A novel stability analysis of linear systems under asynchronous samplings. Automatica, 48(1), 177-182.

Willems, J.C. (1972). Dissipative dynamical systems. part I: general theory. Archive for Rational Mechanics and Analysis, 45(5), 321-351.

Zhang, W., Branicky, M.-S., Phillips, S.-M. (2001). Stability of networked control systems. IEEE Control Systems Magazine, 21(1), 84-99.

Zhou, K., Doyle, J.C., Glover, K. (1996). Robust and optimal control. Vol. 40. New Jersey: Prentice hall. 